\title{
Getting real about biomarkers
}

\section{By Steve Usdin, Washington Editor}

Every week, academic scientists publish dozens of articles on biomarkers. Some results are strong enough to persuade drug companies to use certain biomarkers to guide development decisions. But only a handful of the ones that have been incorporated into marketing applications are accepted by regulators as evidence of efficacy or safety.

Moving biomarkers from academic journals to drug labels is a prerequisite for advancing personalized medicine and for enhancing the overall efficiency of drug development. However, identifying prospective markers has proven far simpler than getting them accepted by regulators.

Potentially useful toxicity tests based on biomarkers get stuck in regulatory purgatory in large part because there has not been a process for determining what kinds of data need to be assembled to convince regulators that the tests are reliable, sensitive and specific predictors of safety or efficacy.

Now, a public-private effort kick-started by a Cooperative Research and Development Agreement (CRADA) between the Food and Drug Administration and Novartis AG has created a roadmap for one step in the process of moving a biomarker from discovery to regulatory acceptance for clinical studies or labeling.

This first pathway applies to FDA qualification of biomarkers for preclinical use. In addition to mapping out the pathway, the CRADA applied it in order to qualify several biomarkers to detect nephrotoxicity, an area in which existing preclinical tests are unable to assess toxicity until kidney damage is irreversible.

The project subsequently was expanded and turned over to the Predictive Safety Testing Consortium (PSTC), a group of drug companies that is being managed by a nonprofit organization, the Critical Path Institute (C-Path). C-Path was founded in 2005 by the FDA, SRI International and the University of Arizona. ${ }^{1}$

The PSTC consists of 15 pharmaceutical companies, ClinXus, which is a nonprofit organization that conducts biomarker R\&D, and predictive toxicology company Iconix Biosciences Inc., a unit of Entelos Inc. (See Table 1, "Predictive Safety Testing Consortium members.")

About 180 scientists from industry are working on five PSTC projects. In addition to nephrotoxicity, which is the most advanced project, the consortium is working on biomarkers for hepatotoxicity, vascular injury, nongenotoxic carcinogenicity and myopathy.

The nephrotoxicity project was clearly aimed at picking low-hanging fruit-there is much room for improvement on measures of kidney damage, a number of candidate markers are readily at hand and testing them is relatively straightforward.

Although none of the PSTC's nephrotoxicity biomarkers is newtotal urinary protein and albumin, for example, have been measured for decades - they cannot currently be used to support drug applications because the work necessary to qualify them for regulatory purposes had never been conducted.

"The CRADA with Novartis kick-started the effort that is going on now in the consortium and provided a blueprint for the pilot biomarker qualification process that we have been setting up at the agency," Felix Frueh, associate director of genomics in the FDA's Office of Clinical Pharmacology and Biopharmaceutics, told SciBX.

New nephrotoxicity biomarkers are needed because the toxicity markers that regulators accept today don't produce signals until injury is irreversible. Frueh noted that a signal from blood urea nitrogen (BUN) or creatinine tests can effectively kill a compound.

"The current markers, BUN and serum creatinine, tell you how dead your kidney is, but are not very useful in assessing a gradual scale of damage," he said.

The new toxicity markers would dramatically increase the sensitivity and specificity of preclinical tests for kidney damage, according to Frueh. This could give industry and regulators confidence to continue the development of compounds in the face of safety signals that, based on older, blunter instruments, would have been showstoppers.

Frueh said the ability to "detect toxicity very, very early, while it is reversible" would make it possible for the FDA to allow clinical research on compounds even if there is some evidence that they could cause

Table 1. Predictive Safety Testing Consortium members.

Abbott Laboratories (NYSE:ABT)

Amgen Inc. (NASDAQ:AMGN)

AstraZeneca plc (LSE:AZN; NYSE:AZN)

Boehringer Ingelheim $\mathrm{GmbH}$

Bristol-Myers Squibb Co. (NYSE:BMY)

ClinXus

Eli Lilly and Co. (NYSE:LLY)

GlaxoSmithKline plc (LSE:GSK; NYSE:GSK)

Iconix Biosciences Inc., part of Entelos Inc. (LSE:ENTL)

Johnson \& Johnson (NYSE:JNJ)

Merck \& Co. Inc. (NYSE:MRK)

Novartis AG (NYSE:NVS; SWX:NOVN)

Pfizer Inc. (NYSE:PFE)

Roche (SWX:ROG)

sanofi-aventis Group (Euronext:SAN; NYSE:SNY)

Schering-Plough Corp. (NYSE:SGP)

Wyeth (NYSE:WYE)

Source: Critical Path Institute 
kidney damage. More sensitive biomarkers also could make it possible for patients to be exposed to drugs that cause kidney damage in some individuals because exposure could be halted at the first sign of toxicity while the injury were still reversible.

Preclinical use of the biomarkers will clearly be helpful for making go or no-go decisions prior to Phase I testing, but nevertheless it is only an interim step toward qualifying biomarkers for clinical uses.

Clinical qualification, which will require studies demonstrating that biomarker results reliably predict clinical outcomes, could support the inclusion of biomarker-based tests on product labels and facilitate the use of biomarkers by physicians to make treatment decisions.

"It is important to make a distinction between using biomarkers for internal decision making in a company for progressing a compound versus being qualified for regulatory decision making," said John Orloff, SVP and head of U.S. drug and medical regulatory affairs at Novartis.

\section{The pathway}

The Novartis-FDA CRADA was designed to answer an important question the FDA did not address in a March 2005 biomarker guidance

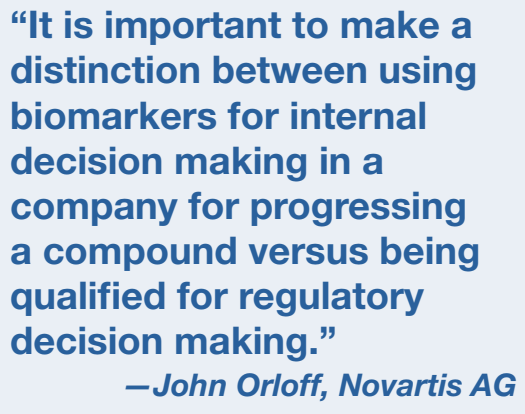

"This is a landmark effort in the sense that existing data for promising markers were evaluated together with regulators, a scientific plan to address information gaps was developed and agreed upon, the experiments were conducted, and the data were analyzed and submitted to regulators with proposals for particular 'fit for use' claims for each marker,' said Kevin Carl, senior associate director of drug regulatory affairs at Novartis.

Scientists at the FDA and EMEA serve as consultants to the PSTC but are not members. Nevertheless, their active involvement is essential for the project's success, Frueh said.

"I don't think industry would be committing millions of dollars to conduct the experiments and generate the datasets needed to qualify biomarkers if there wasn't the recognition that this is going to be a plausible approach from a regulatory view," he told $S c i B X$.

EMEA participation is important, "because you don't want to have biomarkers that are useful in one geographic region and not in another," Frueh said. Japanese regulators have observed PSTC nephrotoxicity biomarker meetings and may review data from the consortium's future submissions, he added.

The selection and cross-validation processes "are all transparent," noted C-Path president document: how to reclassify a "probable" valid biomarker as a "known" valid biomarker. ${ }^{2}$

The document divided valid biomarkers into three categories: exploratory, known and probable. A biomarker is known (acceptable for regulatory purposes) if there is "widespread agreement in the medical or scientific community about the physiologic, toxicologic, pharmacologic, or clinical significance of the results," according to the guidance.

The guidance stated that a valid biomarker may be "probable," and therefore not accepted by regulators, for three main reasons: because "data elucidating its significance may have been generated within a single company and may not be available for public scientific scrutiny," because data on its significance are not conclusive or because data on its performance have not been independently verified.

An exploratory biomarker is a candidate for reclassification as a known or probable valid biomarker based on additional data.

The pathway outlined through the FDA-Novartis CRADA, and refined through the PSTC's work, starts with a request to the agency to qualify a biomarker for a specific use. In response, the FDA recruits a biomarker qualification review team and the biomarker sponsor submits data on the proposed biomarker as a Voluntary Data Submission (VXDS).

Next, the FDA review team and the biomarker sponsor jointly design studies that are required to qualify the biomarker, and the sponsor submits the study results to the review team, which then accepts or rejects the biomarker for specific uses.

\section{First submission}

PSTC's nephrotoxicity biomarker application, which incorporated data from the FDA-Novartis CRADA as well as data from FDA laboratories, is the first joint submission from a consortium to the FDA and the European Medicines Evaluation Agency (EMEA), and it is the first VXDS submission to the FDA, according to William Mattes, C-Path's director of toxicology. and CEO Raymond Woosley. "Everything is available to any scientist who wants to be part of this. We've set up a grand rounds process at FDA where scientists come in and discuss this with other scientists who haven't been involved in it."

\section{Qualifying the biomarkers}

The PSTC working group identified 23 potential preclinical urinary nephrotoxicity biomarkers. Based on advice from the FDA and EMEA about the kinds of data required to qualify the markers, the consortium designed protocols for evaluating their sensitivity and specificity.

Preliminary work included gene expression analysis of kidney and liver tissue, as well as experiments to determine doses of the nephrotoxicants that would produce lesions.

The PSTC adopted a common system for grading lesions on a scale from one to five. In addition, the consortium agreed on terminology for classifying lesion types, as well as a scheme for assigning lesions to specific regions of the kidney.

When the dosing and assessment criteria were agreed, the PSTC began a series of identical experiments in rats.

Experiments were conducted with four doses (control, low, mid and high) of eight nephrotoxicants as well as two hepatotoxicants as controls. Study designs included multiple dose groups and were conducted for different periods of time, up to a maximum of 14 days of dosing (see Table 2, “Toxicity studies”).

In addition to histopathology, the gold standard for preclinical nephrotoxicity, analyses included in-life data such as food consumption and body weight, clinical chemistry including creatinine and BUN, and multiplex ELISA measurements on urine.

Based upon a candidate marker's ability to identify particular types of kidney pathology, and on advice from the FDA and EMEA, the PSTC whittled the 23 potential biomarkers down to 7 that were included in the initial submission, said Mattes. 
Table 2. Toxicity studies. The Predictive Safety Testing Consortium (PSTC) tested the sensitivity and specificity of proposed preclinical nephrotoxicity biomarkers in rats that were exposed to eight nephrotoxicants. In addition, the PSTC conducted identical studies using two hepatotoxicants that do not cause kidney damage: $\alpha$-naphthyisothiocyanate (ANIT) and methapyrilene, to assess the ability of the biomarkers to discriminate between kidney and liver toxicities.

\begin{tabular}{|c|c|c|c|c|}
\hline Nephrotoxicant & Tubule & Glomerulus & Collecting duct & Mode of toxicity \\
\hline Gentamycin & $\mathrm{X}$ & $(\mathrm{X})$ & & Lysosomal phospholipidosis \\
\hline Puromycin & $\mathrm{X}^{(2 \mathrm{nd})}$ & $\mathrm{X}$ & & Damage to podocytes \\
\hline Vancomycin & $\mathrm{X}$ & & & Oxidative stress (free radicals) \\
\hline Doxorubicin & $\mathrm{X}^{(2 \mathrm{nd})}$ & $\mathrm{X}$ & & Oxidative stress to glomerulus filtration membrane \\
\hline Furosemide & $\mathrm{X}$ & & & Mineralization \\
\hline Lithium carbonate & $\mathrm{X}$ & $(\mathrm{X})$ & $\mathrm{X}$ & Influences formation of intracellular cyclic adenosine monophosphate \\
\hline Cisplatin & $\mathrm{X}$ & $(\mathrm{X})$ & $(\mathrm{X})$ & Direct DNA alkylation (oxidative stress) \\
\hline Tacrolimus & $\mathrm{X}$ & $(\mathrm{X})$ & & Complex (vasoconstriction, calcification) \\
\hline
\end{tabular}

$\mathrm{X}$ signifies that the agent is directly toxic to the particular renal structure. $(X)$ signifies that the agent may be directly toxic to that particular renal structure, but either to a lesser extent than a structure marked with $X$ or only under certain conditions. $X^{(2 n d)}$ signifies that the agent is indirectly toxic to that particular renal structure (that is, its effects on primary renal structure result in toxicity to the secondary renal structure). Source: Novartis $A G$ presentation to the Institute of Medicine

Novartis contributed some of the seven markers and PSTC member Merck \& Co. Inc. contributed others. Some were proposed by both companies, according to Mattes.

The consortium may submit other kidney toxicity biomarkers from the list to the FDA and EMEA in the future, according to Carl (see Table 3, "Kidney toxicity biomarkers").

Preliminary PSTC data presented by Novartis at an Institute of Medicine meeting in April 2007 illustrated the sensitivity of some of the submitted biomarkers.

Using cisplatin, a chemotherapeutic that is known to cause serious kidney damage, Novartis reported that creatinine was elevated above the control threshold only when $3 \mathrm{mg} / \mathrm{kg}$ of drug, the highest dose, was administered; lower doses did not produce a toxicity signal based on creatinine.

Moreover, the creatinine test detected only grade three lesions, indicative of the worst kidney injury.

In contrast, Kim-1, a protein that is undetectable in healthy kidneys but is present in those that have suffered ischemic or toxic injury, was sensitive to a lower dose and to less-severe lesions. The Kim-1 marker was detected in rats exposed to $1 \mathrm{mg} / \mathrm{kg}$ of cisplatin, the middle dose, and histopathology examination determined that it was associated with grade one and two lesions. In addition to increased sensitivity, many of the submitted biomarkers signal damage to specific parts of the kidney. For example, urinary cystatin $\mathrm{C}$ predicts glomerular alteration, whereas clusterin signals distal and proximal tubule damage.

\section{Regulatory decisions}

The PSTC submitted its nephrotoxicity application to the FDA and EMEA on June 12,2007. The FDA will notify the PSTC of its decisions "very soon," according Frueh.

An EMEA decision on the biomarker qualification applications is also "imminent," according to Novartis.
The FDA is clearly impressed by both the results and the process used to obtain them. "The data are outstanding; there is no argument over how useful it is going to be," Frueh told SciBX.

Following FDA and EMEA approval of the PSTC submission, the consortium's next steps could include further qualification of other kidney biomarkers, probably involving clinical studies, and greater integration of clinical and preclinical data, Mattes said.

This summer, Novartis plans to submit additional proposed preclinical genomic and serum biomarkers of nephrotoxicity that are not part of the PSTC submission, Carl said. Novartis and the PSTC also plan to begin work to qualify some of the tests as valid clinical biomarkers.

"Because of the promise the new markers have shown in the data already generated, further translational studies are planned by Novartis and other members of the PSTC in order to generate the needed data to eventually have the markers qualified for broad clinical use," Carl told SciBX.

From the FDA's point of view, qualification of preclinical markers is analogous to approving an

IND, according to Freuh.

Approval of the PSTC application "will mean that now we have investigational biomarkers," he said. "That is not the same as saying they should be used broadly in clinical trials-we aren't there yet, but clearly this is where we want to go."

Qualification as clinical biomarkers could be based on studies with drugs like cisplatin that are nephrotoxic but are not fatal, according to Frueh. "You can imagine straightforward experiments where a biomarker was measured in people who take drugs in order to assess the level of toxicity", he said.

Successful demonstration of the utility of the biomarkers in a clinical trial, for example using them to detect kidney damage early enough to stop exposure to a drug before it causes permanent kidney damage, could lead to approval of their inclusion on a drug label, Frueh said. 
Table 3. Kidney toxicity biomarkers. The Critical Path Institute's Predictive Safety Testing Consortium (PSTC) has studied 23 urinary nephrotoxicty biomarkers. In July 2007, it submitted seven to the FDA and EMEA for qualification as preclinical biomarkers, shown in the first list below, and may submit others in the future. Histopathology studies demonstrated associations between many of the biomarkers and damage to specific areas of the kidney (glomerulus, proximal tubule, distal tubule, loop of henle and collecting duct).

\begin{tabular}{|c|c|c|}
\hline Submitted biomarkers & Area of kidney & Description \\
\hline Albumin & Not applicable & Protein found in blood plasma and urine that transports small molecules \\
\hline$\beta 2$-microglobulin & Glomerulus; proximal tubule & $\begin{array}{l}\text { Low molecular weight protein that is eliminated by glomerular filtration but } \\
\text { completely reabsorbed by the tubules }\end{array}$ \\
\hline Clusterin & Distal tubule; proximal tubule & Highly conserved protein associated with apoptosis \\
\hline Cystatin C & Glomerulus & $\begin{array}{l}\text { Nonglycosylated protein found in all nucleated cells; inhibitor of the elastin- } \\
\text { and collagen-degrading cysteine proteases }\end{array}$ \\
\hline Kim-1 & Proximal tubule & Membrane protein implicated in damage and repair \\
\hline Trefoil factor 3 & Not applicable & $\begin{array}{l}\text { Protein secreted onto mucosal surfaces; has tissue-specific activity including } \\
\text { inhibiting apoptosis and modulating motogenic activity }\end{array}$ \\
\hline Total urinary protein & Not applicable & Amount of protein found in urine \\
\hline Other biomarkers studied & Area of kidney & Description \\
\hline Calbindin $\mathrm{d} 28$ & Collecting duct; distal tubule & Vitamin D-dependent calcium binding protein \\
\hline Epidermal growth factor & Distal tubule; proximal tubule & $\begin{array}{l}\text { Growth factor that plays a role in cell system regeneration and acute renal } \\
\text { injury recovery }\end{array}$ \\
\hline Glutathione S-transferase- $\alpha$ & Proximal tubule & Cystolic detoxification enzyme \\
\hline Glutathione S-transferase- $\mu$ & Distal tubule & Cystolic detoxification enzyme \\
\hline Interferon- $\gamma$-induced $10 \mathrm{kDa}$ protein & Not applicable & Chemokine that mediates the proliferation of human mesangial cells (HMC) \\
\hline $\begin{array}{l}\text { Lipocalin } 2 \text { (neutrophil gelatinase- } \\
\text { associated lipocalin; NGAL) }\end{array}$ & Proximal tubule & Transporter protein that is upregulated during inflammation \\
\hline Macrophage migration inhibitory factor & Not applicable & $\begin{array}{l}\text { Tautomerase that is involved in development of septic shock, arthritis and } \\
\text { glomerulonephritis }\end{array}$ \\
\hline Monokine induced by interferon- $\gamma$ & Not applicable & Chemokine chemoattractant for activated $\mathrm{T}$ cells \\
\hline $\mathrm{N}$-Acetyl- $\beta$-D-glucosaminidase & Proximal tubule & Lysosomal enzyme \\
\hline Osteoactivin & Not applicable & Cell-surface and lysosomal protein involved in bone matrix production \\
\hline Osteopontin & $\begin{array}{l}\text { Distal tubule; loop of henle; proximal } \\
\text { tubule }\end{array}$ & $\begin{array}{l}\text { Acidic protein that binds to hydroxylapatite and is implicated in mediating the } \\
\text { binding of osteoclasts to the mineral matrix of bone surfaces }\end{array}$ \\
\hline Podocin & Glomerulus & Slit diaphragm protein that binds the cytoplasmic domain of nephrin \\
\hline Replication protein A & Not applicable & Protein involved in DNA repair \\
\hline Tissue inhibitor of metalloproteinase-1 & Distal tubule; proximal tubule & Metalloproteinase inhibitor involved in the regulation of bone modeling \\
\hline Uromodulin (Tamm-Horsfall) & Loop of henle & Glycoprotein with potential role in regulating cytokines \\
\hline VEGF & Not applicable & Polypeptide that stimulates new blood vessel formulation \\
\hline
\end{tabular}

Source: Critical Path Institute and Novartis AG

The markers "are assessments at the time damage occurs," he noted, but the FDA might consider them "predictive in the sense that if there is continued exposure, you would expect these markers to go up further."

Frueh said the ability to detect kidney injury while it is reversible could make it possible to salvage drugs that have been shelved because of fears they cause irreversible damage. The goal is "to be able to use this type of information to move compounds that are on hold, are stuck, or have been discontinued because of preclinical toxicity," he said.

"Preclinical qualification is the necessary first step," Frueh concluded. "We hope the next step will demonstrate the usefulness of these markers in the clinical context. Then we will have true translational markers."

\section{REFERENCES}

1. Usdin, S. \& Maggos, C. BioCentury 13(50), A1-A5; Nov. 14, 2005

2. U.S. Food and Drug Administration. Guidance for industry, pharmacogenomic data submissions. <http://www.fda.gov/cber/gdlns/ pharmdtasub.pdf> (March 31, 2005)

\section{COMPANIES AND INSTITUTIONS MENTIONED}

ClinXus, Grand Rapids, Mich.

Critical Path Institute, Tucson, Ariz. and Rockville, Md.

Entelos Inc. (LSE:ENTL), Foster City, Calif.

European Medicines Evaluation Agency, London, U.K.

Food and Drug Administration, Rockville, Md.

Iconix Biosciences Inc., Mountain View, Calif.

Institute of Medicine, Washington, D.C.

Novartis AG (NYSE:NVS; SWX:NOVN), Basel, Switzerland

Merck \& Co. Inc. (NYSE:MRK), Whitehouse Station, N.J.

SRI International, Menlo Park, Calif.

University of Arizona, Tucson, Ariz. 